\title{
How agents see things: \\ On visual representations in an emergent language game
}

\author{
Diane Bouchacourt \\ Facebook AI Research \\ dianeb@fb. com
}

\author{
Marco Baroni \\ Facebook AI Research \\ mbaronieflo. com
}

\begin{abstract}
There is growing interest in the language developed by agents interacting in emergentcommunication settings. Earlier studies have focused on the agents' symbol usage, rather than on their representation of visual input. In this paper, we consider the referential games of Lazaridou et al. (2017), and investigate the representations the agents develop during their evolving interaction. We find that the agents establish successful communication by inducing visual representations that almost perfectly align with each other, but, surprisingly, do not capture the conceptual properties of the objects depicted in the input images. We conclude that, if we are interested in developing language-like communication systems, we must pay more attention to the visual semantics agents associate to the symbols they use.
\end{abstract}

\section{Introduction}

There has recently been a revival of interests in language emergence simulations involving agents interacting in visually-grounded games. Unlike earlier work (e.g., Briscoe, 2002; Cangelosi and Parisi, 2002; Steels, 2012), many recent simulations consider realistic visual input, for example, by playing referential games with real-life pictures (e.g., Jorge et al., 2016; Lazaridou et al., 2017; Havrylov and Titov, 2017; Lee et al., 2018; Evtimova et al., 2018). This setup allows us to address the exciting issue of whether the needs of goal-directed communication will lead agents to associate visually-grounded conceptual representations to discrete symbols, developing naturallanguage-like word meanings. However, while most studies present some analysis of the agents' symbol usage, they pay little or no attention to the representation of the visual input that the agents develop as part of their evolving interaction.

We study here agent representations following the model and setup of Lazaridou et al. (2017).
This is an ideal starting point, since it involves an extremely simple signaling game (Lewis, 1969), that is however played with naturalistic images, thus allowing us to focus on the question of how the agents represent these images, and whether such representations meet our expectations for natural word meanings. In their first game, Lazaridou's Sender and Receiver are exposed to the same pair of images, one of them being randomly marked as the "target". The Sender always sees the target in the left position, and it must pick one discrete symbol from a fixed vocabulary to send to the Receiver. The Receiver sees the images in random order, together with the sent symbol, and it tries to guess which image is the target. In case of success, both players get a payoff of 1 . Since an analysis of vocabulary usage brings inconclusive evidence that the agents are using the symbols to represent natural concepts (such as beaver or bayonet), Lazaridou and colleagues next modify the game, by presenting to the Sender and the Receiver different images for each of the two concepts (e.g., the Sender must now signal that the target is a beaver, while seeing a different beaver from the one shown to the Receiver). This setup should encourage conceptlevel thinking, since the two agents should not be able to communicate about low-level perceptual characteristics of images they do not share. Lazaridou and colleagues present preliminary evidence suggesting that, indeed, agents are now developing conceptual symbol meanings. We replicate Lazaridou's games, and we find that, in both, the agents develop successfully aligned representations that, however, are not capturing conceptual properties at all. In what is perhaps our most striking result, agents trained in either version of the game succeed at communicating about pseudoimages generated from random noise (Fig. 2). We conclude that, if we want interactive agents to 
develop a vocabulary of words denoting natural meanings, more attention must be paid to the way in which they are representing their perceptual input.

\section{Experimental setup}

Architecture We re-implement Lazaridou's Sender and Receiver architectures (using their better-behaved "informed" Sender). Both agents are feed-forward networks. The Sender takes image representations as input, it projects them into its own representational space, compares them, and finally outputs a probability distribution over vocabulary symbols, from which a single discrete symbol is then sampled. We report here results obtained with an output vocabulary of 100 symbols, but the same patterns were observed using a range of sizes from 2 to 1,000 . The Receiver takes as input the target and distractor input image representations in random order, as well as the symbol produced by the sender (as a vocabulary-sized one-hot vector). It embeds the images and the symbol into its own representational space, where it performs a symbol-to-image comparison, producing a probability distribution over the two images, one of which is selected by sampling from this distribution. If the Receiver selected the target image, a reward of 1 is assigned to both agents. The whole architecture is jointly trained by letting the agents play, and updating their parameters with Reinforce (Williams, 1992). See Lazaridou et al. (2017) for details.

Data Following Lazaridou et al. (2017), for each of the 463 concepts they used, we randomly sample 100 images from ImageNet (Deng et al., 2009). We construct 50,000 mini-batches of 32 image pairs during training and 1,024 pairs for validation. We construct a held-out test set in the same way by sampling 10 images per concept from ImageNet (for 2 concepts, we were not able to assemble enough further images), for a total of 4,610 . We compute RSA scores (see below) on the crossproduct of these images. We also use the heldout set to construct mini-batches of images pairs to compute test performance. Following Lazaridou, the images are passed through a pre-trained VGG ConvNet (Simonyan and Zisserman, 2015). The input vector fed to the agents is the second-tolast 4096-D fully connected layer ${ }^{1}$.

\footnotetext{
${ }^{1}$ We found very similar results with the top 1000 -D softmax layer.
}

Games We re-implement both Lazaridou's same-image game, where Sender and Receiver are shown the same two images (always of different concepts), and their different-image game, where the Receiver sees different images than the Sender's. We repeat all experiments using 100 random initialization seeds. As we faithfully reproduced the setup of Lazaridou et al. (2017), we refer the reader there for hyper-parameters and training details.

\section{Experiments}

We first asked in which way playing the game affects the way agents "see" the input data, that is, in which way their image embeddings differ from the input image representations, and from each other. Concerning Sender and Receiver, a reasonable expectation is that successful communication implies a convergence of representations. How should these representations relate to the input? Recall that input representations are from one of the top layers of a state-of-the-art ConvNet trained on ImageNet concept categorization, and the top layers of such networks are known to capture highlevel concept semantics (Zeiler and Fergus, 2014). The game image pairs are always sampled from different concepts. So, it would make sense for the agents to simply learn to carry through the similarity structure of the input space, in order to communicate about distinct concepts. Consequently, we predicted that, as training proceeds, Sender and Receiver representations will become closer to each other, and to the input ones.

In order to compare the similarity structure of input, Sender and Receiver spaces, we borrow representational similarity analysis (RSA) from computational neuroscience (Kriegeskorte et al., 2008). Given two sets $r_{1}$ and $r_{2}$ of representations of the same item collection (e.g., $r_{1}$ is the collection of input images mapped in Sender embedding space and $r_{2}$ is the same collection represented by Receiver), we first compute $s_{1}$ as all possible pairwise (cosine) similarities between the representations in $r_{1}$, and $s_{2}$ as those in $r_{2}$. We then compute the (Spearman) correlation between the similarity vectors $s_{1}$ and $s_{2}$. This latter value, which we will call RSA score, measures the global agreement between $s_{1}$ and $s_{2}$, relative to the chosen input collection. If $N$ is the number of items in the collection that we compute representations for, both similarity vectors $s_{1}$ and $s_{2}$ are of length 


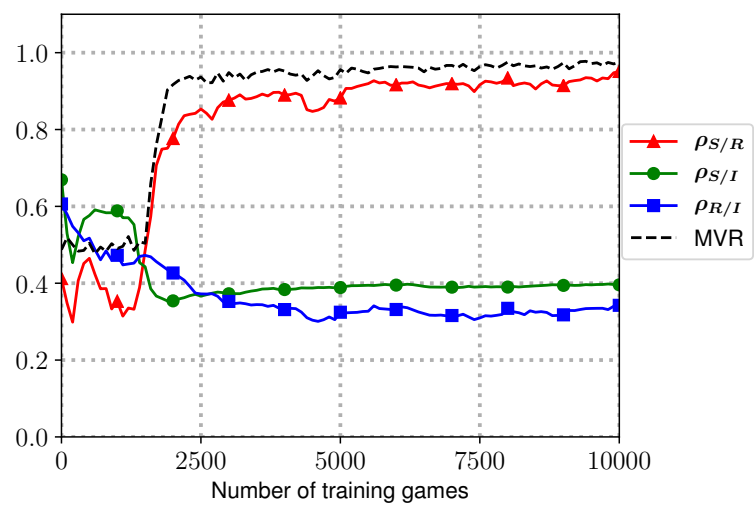

Figure 1: RSA scores of the two agents $\left(\rho_{S / R}\right)$, and of each agent with the input $\left(\rho_{S / I}\right.$ and $\left.\rho_{R / I}\right)$, during the first 10,000 training games. $S$ refers to Sender, $R$ to Receiver, $I$ to input. Values remain stable until end of training. Best viewed in color.

$N(N-1)$. Therefore, it is not necessary for representations $r_{1}$ and $r_{2}$ to belong to the same space (for example, in our case, input and agent vectors have different dimensionality).

Figure 1 shows RSA and mean validation reward (MVR) development curves for the crossvalidated best seed in the same-image game. At the beginning of training, the RSA scores are nonzeros, which is expected as the two agents architectures are similar and randomly initialized the same way. They are also somewhat correlated with the input, which we attribute to the fact that untrained neural networks can already extract relevant image features (Jarrett et al., 2009). As training converges, Sender and Receiver similarity spaces also converge. However, contrary to our prediction, the agent similarity spaces are not strongly correlated with the input visual space. We note that, during the first few hundred games, the Sender (green curve) aligns with the input, but the Receiver (blue curve) does not. Therefore, it seems that, in order to establish communication, the two agents have to drift from the input. Indeed, when communication is successfully established at the end of training, ${ }^{2}$ the two agents have a RSA score of $\rho_{S / R}=0.98$. However either agent's score with the input is a much lower $\rho_{S / I}=\rho_{R / I}=0.33 .{ }^{3}$ On the contrary, when the agents fail to establish communication, by the end of training their RSA score is just $\rho_{S / R}=0.39$, but they stay closer to the input $\left(\rho_{S / I}=0.58\right.$ and

\footnotetext{
${ }^{2}$ We consider training successful if MVR $\geq 80 \%$.

${ }^{3}$ Values averaged over the 96 successful seeds.
}

$\left.\rho_{R / I}=0.42\right){ }^{4}$

The drift of the agents from input similarity could be attributed to the characteristics of the game they are playing. Since they are only asked to distinguish between pictures of different concepts, they have no incentive to keep different instances of a concept distinct (if the agents are never asked to distinguish one dog from another, they might eventually become unable to tell dogs apart). That is, we might be assisting to the inception of a form of categorical perception (Goldstone and Hendrickson, 2010), whereby the agents lose sensitivity to within-category differences. If this is the case, we should observe that sameconcept image similarity is higher in Sender (or Receiver) space with respect to input space. However, this turns out not to be the case. To the contrary, average pairwise same-concept similarity is consistently lower in Sender space than in the input (mean $z$-normalized same-concept similarity in input space is at 1.94 vs. 0.57 in Sender space, averaged across successful seeds). A similar effect is observed by looking at higher-class (mammal, furniture, etc.) similarities: images from the same classes become less similar in Sender space (0.61 $z$-normalized within-class input similarity vs. 0.30 in Sender space). This suggests that the agents are becoming less proficient at capturing the similarity among instances of the same concept or of the same class. The same conclusion is qualitatively supported by the pairs of images that underwent the largest shift between input and Sender space. For example, for two test images of avocados which have an input similarity of 0.82 (and are reasonably similar to the human eye), the Sender similarity is at the low value of -0.27 (Receiver similarity is -0.59). Contrarily, for an image of a cabin in a field and an image of a telephone that have an intuitively correct very low input similarity of 0.02 , the Sender similarity for these images is 0.94 (Receiver similarity is 0.95 ).

Lazaridou et al. (2017) designed their second game to encourage more general, concept-like referents. Unfortunately, we replicate the anomalies above in the different-image setup, although to a less marked extent. When successful communication is established at the end of training, the agents have $\rho_{S / R}=0.90$. But again, the agents' representation do not align with the input space: their scores with the input are at lower values of $\rho_{S / I}=$

\footnotetext{
${ }^{4}$ Values averaged over the 4 failing seeds.
} 


\begin{tabular}{|c|cccc|}
\hline & \multicolumn{4}{|c|}{ Test } \\
\hline & & Same im. & Diff. im. & Noise \\
哥 & Same im. & 100 & 72 & 95 \\
& Diff. im. & 98 & 83 & 87 \\
\hline
\end{tabular}

Table 1: Percentage average rewards on the sameimage, different-image and noise test sets for agents trained in the same- and different-image games (chance level at 50\%). For each game, values are averaged on 10 test runs consisting of 1, 000 games of mini-batches of 32 image pairs, using the cross-validated best seed.

0.40 and $\rho_{R / I}=0.37 .^{5}$ In case of communication failure, by the end of training their RSA score is at the lower value of $\rho_{S / R}=0.74$, and their values with respect to the input are $\rho_{S / I}=0.36$ and $\rho_{R / I}=0.34 .^{6}$ Again, same-concept images drift apart in agent space, although now to a lesser extent (1.94 $z$-normalized mean similarity in input space vs. 1.07 in Sender space). More encouragingly, we don't find the same pattern for withinclass mean similarities (0.61 input space vs. 0.75 Sender space).

We must conjecture that the agents are comparing low-level properties of the image pairs, independently of the game they play. As an extreme way to test this, we look at how agents trained to play the two games behave when tested with input pairs that are just random noise vectors drawn from a standard Normal distribution. ${ }^{7}$ If the agents are indeed indifferent to the objects represented by images, the radical shift in the nature of the input to the game should not affect them much.

Results are shown in Table 1. We confirm that the same-image game is the easiest, and we observe that agents trained in one game perform reasonably well on the other. More importantly, no matter which game they are trained on, the agents perform very well on noise input! This confirms our hypothesis that the Sender and Receiver are able to communicate about input data that contain no conceptual content at all, which in turn suggests that they haven't extracted any concept-level information (e.g., features that would allow them to recognize instances of the $d o g$ or chair category) during training. To get a sense of the sort of noise pairs agents succeed to communicate about, Fig-

\footnotetext{
${ }^{5}$ Values averaged over the 19 successful seeds.

${ }^{6}$ Values averaged over the 81 failing seeds.

${ }^{7}$ As during training inputs are divided by their norm, we also normalize each noise vector, so multiple noise variances would have no effect.
}
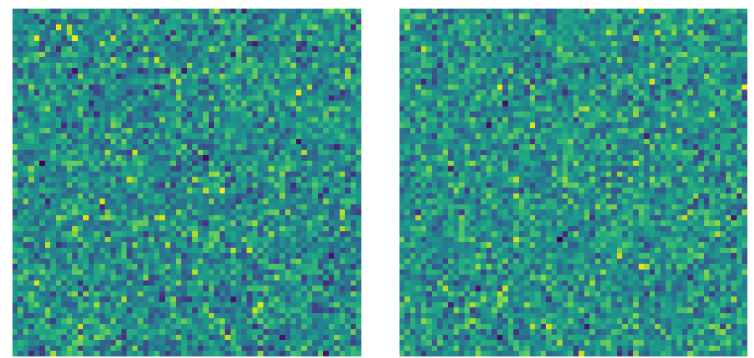

Figure 2: Noise vectors agents trained on the sameimage game successfully communicate about.

ure 2 provides an example.

Finally, we draw 1,000 noise pairs $\left(z_{1}, z_{2}\right)$, and present each to the Sender with either $z_{1}$ or $z_{2}$ as target. We then compare, pair by pair, whether the highest probability symbol changes when the target is swapped. We average across 10 random runs using the best cross-validated seed. In both versions of the game, for more than $99 \%$ of the pairs, the symbol with highest probability changes when the target is swapped. This suggests that the agents perform a relative comparison of the two inputs, rather than an absolute one, in line with the general conclusion that they are not using the vocabulary to denote stable conceptual properties of the objects depicted in the images.

\section{Discussion}

Existing literature in game theory already showed that convergence towards successful communication is ensured under specific conditions (see Skyrms (2010) and references therein). However, the important contribution of Lazaridou et al. (2017) is to play a signaling game with real-life images instead of artificial symbols. This raises new empirical questions that are not answered by the general mathematical results, such as: When the agents do succeed at communicating, what are the input features they rely upon? Do the internal representations they develop relate to the conceptual properties of the input? Our study suggests that the agents' representations are not capturing general conceptual properties of different objects, but they are rather specifically tuned to successfully distinguish images based on inscrutable lowlevel relational properties.

Interestingly, our conclusions can be aligned with findings in psycholinguistic experimental literature on dialogue. In order to achieve communication, the agents develop a form of "conceptual 
pact" (Brennan and Clark, 1996): Their internal representations align while at the same time drifting away from human-level properties of the input. The agents agree on a shared use of the vocabulary, that does not correspond to concepts in the input data.

In future work, we would like to encourage the development of more natural word meanings by enforcing the agent representations to stay more faithful to the perceptual input they receive. Moving ahead, it is fundamental to design setups where agents would have stronger reasons to develop human-like communication strategies.

\section{Acknowledgments}

We thank Angeliki Lazaridou, Douwe Kiela and Calvin Lee for their useful discussions and insights. We also thank Francisco Massa for his help on setting up the experiments.

\section{References}

Susan E. Brennan and Herbert H. Clark. 1996. Conceptual pacts and lexical choice in conversation. Journal of Experimental Psychology: Learning, Memory, and Cognition, 22:1482-1493.

Ted Briscoe, editor. 2002. Linguistic evolution through language acquisition. Cambridge University Press, Cambridge, UK.

Angelo Cangelosi and Domenico Parisi, editors. 2002. Simulating the evolution of language. Springer, New York.

Jia Deng, Wei Dong, Richard Socher, Lia-Ji Li, and Li Fei-Fei. 2009. Imagenet: A large-scale hierarchical image database. In Proceedings of CVPR, pages 248-255, Miami Beach, FL.

Katrina Evtimova, Andrew Drozdov, Douwe Kiela, and Kyunghyun Cho. 2018. Emergent communication in a multi-modal, multi-step referential game. In Proceedings of ICLR Conference Track, Vancouver, Canada. Published online: https://openreview. net/group? id=ICLR. cC/2018/Conference.

Robert Goldstone and Andrew Hendrickson. 2010. Categorical perception. Wiley Interdisciplinary Reviews: Cognitive Science, 1(1):69-78.

Serhii Havrylov and Ivan Titov. 2017. Emergence of language with multi-agent games: Learning to communicate with sequences of symbols. In Proceedings of NIPS, pages 2149-2159, Long Beach, CA, USA.
Kevin Jarrett, Koray Kavukcuoglu, Marc'Aurelio Ranzato, and Yann LeCun. 2009. What is the best multistage architecture for object recognition? In Proceedings of the 12th International Conference on Computer Vision, pages 2146-2153.

Emilio Jorge, Mikael Kågebäck, and Emil Gustavsson. 2016. Learning to play Guess Who? and inventing a grounded language as a consequence. In Proceedings of the NIPS Deep Reinforcement Learning Workshop, Barcelona, Spain. Published online: https://sites.google.com/site/ deeprlnips2016/.

Nikolaus Kriegeskorte, Marieke Mur, and Peter Bandettini. 2008. Representational similarity analysis: Connecting the branches of systems neuroscience. Frontiers in Systems Neuroscience, 2(4):1-28.

Angeliki Lazaridou, Alexander Peysakhovich, and Marco Baroni. 2017. Multi-agent cooperation and the emergence of (natural) language. In Proceedings of ICLR Conference Track, Toulon, France. Published online: https://openreview. net/ group?id=ICLR.cC/2017/conference.

Jason Lee, Kyunghyun Cho, Jason Weston, and Douwe Kiela. 2018. Emergent translation in multi-agent communication. In Proceedings of ICLR Conference Track, Vancouver, Canada. Published online: https://openreview. net/group? id=ICLR.CC/2018/Conference.

David Lewis. 1969. Convention. Harvard University Press, Cambridge, MA.

Karen Simonyan and Andrew Zisserman. 2015. Very deep convolutional networks for large-scale image recognition. In Proceedings of ICLR Conference Track, San Diego, CA. Published online: http://www.iclr.cc/doku.php?id= iclr2015:main.

Brian Skyrms. 2010. Signals: Evolution, learning, and information. Oxford University Press, Oxford, UK.

Luc Steels, editor. 2012. Experiments in Cultural Language Evolution. John Benjamins, Amsterdam.

Ronald Williams. 1992. Simple statistical gradientfollowing algorithms for connectionist reinforcement learning. Machine learning, 8(3-4):229-256.

Matthew Zeiler and Rob Fergus. 2014. Visualizing and understanding convolutional networks. In Proceedings of ECCV (Part 1), pages 818-833, Zurich, Switzerland. 